\section{CSF CYTOKINE AND CHEMOKINE PROFILES IN JAPANESE PATIENTS WITH WEST SYNDROME}

\section{G. Yamanaka}

\section{Department of Pediatrics, Tokyo Medical University, Tokyo, Japan}

Background and aims: We assessed the cytokine and chemokine profiles of cerebrospinal fluid (CSF) in patients with West syndrome to elucidate whether immunological processes are concerned with the pathophysiology of West syndrome.

Methods: We analyzed the CSF levels of Japanese ten patients with West syndrome (WS), eight Influenza virus-associated encephalopathy (IE), as a representative disease with high cytokines storms and twelve controls (cont). All samples of CSF were obtained the first 24 hours after the tonic spasms with informed consent. Seventeen items were measured using the Bio-Plex Multiplex Cytokine Assay, Interleukin (IL)-1 $\beta$, IL-2, IL-4, IL-5, IL-6, IL-7, IL-8, IL-10, IL-12, IL-13, IL-17, G-CSF, GMCSF, INF- $\gamma$, MCP-1, MIP-1 $\beta$ and TNF- $\alpha$. Statistical differences between three independent groups were tested using the nonparametric Kruskal-Wallis test followed by paired testing using the Mann-Whitney test. A $p$ value of 0.05 was considered significant. Results: All of the CSF of cytokine and chemokine levels in the WS and IE groups was significantly higher than those in the cont group. The CSF levels of IL-6, IL-8, G-CSF, INF-y and TNF- $\alpha$ in the WS group were significantly lower than those in the IE group. No significant differences of the other cytokines were detected between the WS and IE groups.

Conclusions: Our results demonstrate that the increased CSF levels of cytokine and chemokine profiles in patients with West syndrome. A larger study should be conducted to clarify which cytokines are mainly concerned with the pathophysiology of WS.

\section{CURRENT COOLING PRACTICE IN THE UK}
D. Azzopardi' ${ }^{1}$ B. Strohm², UK TOBY Cooling Register

${ }^{1}$ Imperial College London, London, ${ }^{2}$ National Perinatal Epidemiology Unit, University of Oxford, Oxford, UK

Background and aims: The use of therapeutic hypothermia to treat neonatal encephalopathy has steadily expanded outside of clinical trial settings. The UK TOBY Cooling Register audits the use of this evolving treatment. In addition to setting standards and providing the means to report complications, the Register accumulates evidence to endorse existing practice, amend guidelines, map services and document demand.

Methods: Cooling centres provide information about their facilities and anonymised clinical data on each cooled baby (resuscitation data, encephalopathy scores, rectal temperatures, clinical complications, outcome information, MRI findings). Two year outcome data are also requested to record the neurodevelopment of cooled babies.

Results: 671 infants born at a median of 40 (IQR 38.4 - 41.1) weeks' gestation and weighing a mean of $3368 \mathrm{~g}$ (SD 680g) were studied. Mean (SD) rectal temperature from 6-72 hrs of the cooling period was $33.5^{\circ} \mathrm{C}(0.61) .23 \%$ of infants died before discharge. MRI findings were reported on $62 \%$ of infants, findings were consistent with hypoxia-ischaemia in most cases. Two year outcome data are less well reported; out of 43 cases, 4 (9\%) infants died before 2 years and $8(20 \%)$ had GMFCS level 3-5.

Conclusions: Cooling has become established as an apparently safe treatment which may improve the outcomes of many infants with neonatal encephalopathy. Surveillance of longer term outcomes is essential in order to ascertain its full potential as a treatment that could provide benefit into childhood and beyond. 